\title{
Use of Guseinov's One-Center Expansion Formulae and Löwdin $\alpha$ Radial Function in Calculation of Two-Center Overlap Integrals over Slater Type Orbitals with Noninteger Principal Quantum Numbers
}

\author{
B.A. Mamedov And E. ÇOPURoĞLU
}

Department of Physics, Faculty of Arts and Sciences, Gaziosmanpaşa University, Tokat, Turkey

(Received May 4, 2010; in final form June 24, 2010)

An efficient calculation algorithm is presented for two-center overlap integrals over noninteger $n^{*}$ Slater type orbitals in molecular coordinate system based on the use of Guseinov's one-center expansion formulae and Löwdin $\alpha$ radial function. These integrals are expressed in terms of overlap integrals of integer $n$ Slater type orbitals. The analytical formulae offer the advantage of direct and efficient calculation of the two-center overlap integrals over noninteger $n^{*}$ Slater type orbitals without the use of numerical methods. Several numerical results obtained are presented to demonstrate the improvements in convergence rates.

PACS: 31 15.-p, 31 15.ae

\section{Introduction}

The two-center overlap integrals over integer $n^{*}$ Slater type orbitals (ISTOs)and noninteger $n^{*}$ Slater type orbitals (NISTOs) have played a major role in evaluation of multicenter multielectron integrals and in electronic structure calculations of molecules. In literature, several types of atomic orbitals have been proposed $[1,2]$. The two more commonly used are the Slater type orbitals (STOs) and Gaussian type orbitals (GTOs). The GTOs have two major disadvantages: (1) They do not have a cusp on the nucleus. (2) They fall off too rapidly for large $r[3,4]$. The STOs and GTOs are not orthogonal with respect to the principal quantum numbers that creates some difficulties arising in the solution of different atomic and molecular problems when the HartreeFock-Roothaan (HFR) and explicitly correlated theories are employed. Thus, the necessity for using the complete orthonormal sets of $\Psi^{\alpha}$-exponential type orbitals ( $\Psi^{\alpha}$-ETOs, $\alpha=1,0,-1,-2, \ldots$ ) introduced by Guseinov as basis functions arises [5]. The STOs and Guseinov's $\Psi^{\alpha}$-ETOs are able to satisfy the cusp condition at the nuclei $[3]$ and decrease exponentially at the large distances [4]. In Refs. [6, 7], general analytical formulae have been obtained to express the overlap integrals with integer and noninteger $n$ STOs in terms of the Guseinov auxiliary function $Q_{n s}^{q}$. Formulae for one-electron two-center molecular integrals over STOs have been introduced in Refs. $[8,9]$ which are used in calculation of integer principal quantum numbers.

In this study, we present a new analytical algorithm for two-center overlap integrals over NISTOs on the basis of Guseinov's one-center expansion formulae and Löwdin $\alpha$ radial function $[5,10]$. It should be noted that the accurate evaluation of the two-center overlap integrals over NISTOs gives the well molecular structure information needed to evaluate the HFR equation. A numerical example is presented to demonstrate the efficiency of the proposed algorithm for arbitrary values of integer and noninteger principal quantum numbers and screening constants of ISTOs and NISTOs and internuclear distances.

\section{Definitions and basic formulae}

In order to evaluate the two-center overlap integrals over NISTOs, we use Guseinov's formula for the one-center expansion of NISTOs in terms of ISTOs defined as $[5,11]$

$$
\chi_{n^{*} l m}(\zeta, \boldsymbol{r})=\lim _{N \rightarrow \infty} \sum_{n^{\prime}=l+1}^{N} V_{n^{*} l, n^{\prime} l}^{\alpha N} \chi_{n^{\prime} l m}(\zeta, \boldsymbol{r}),
$$

where $\alpha=1,0,-1,-2, \ldots$ Here, the normalized NISTOs $\chi_{n * l m}(\zeta, \boldsymbol{r})$ and ISTOs $\chi_{n^{\prime} l m}(\zeta, \boldsymbol{r})$ and expansion coefficients $V^{\alpha N}$ are determined by

$$
\begin{aligned}
& \chi_{n^{*} l m}(\zeta, \boldsymbol{r})=(2 \zeta)^{n^{*}+\frac{1}{2}}\left[\Gamma\left(2 n^{*}+1\right)\right]^{-\frac{1}{2}} \\
& \quad \times r^{n^{*}-1} \mathrm{e}^{-\zeta r} S_{l m}(\theta, \varphi), \\
& \chi_{n l m}(\zeta, \boldsymbol{r})=(2 \zeta)^{n+\frac{1}{2}}[(2 n) !]^{-\frac{1}{2}} \\
& \quad \times r^{n-1} \mathrm{e}^{-\zeta r} S_{l m}(\theta, \varphi), \\
& V_{n^{*} l, n^{\prime} l}^{\alpha N}=\sum_{n^{\prime \prime}=l+1}^{N} \Omega_{n^{\prime} n^{\prime \prime}}^{\alpha l}(N)
\end{aligned}
$$




$$
\begin{aligned}
& \times \frac{\Gamma\left(n^{*}+n^{\prime \prime}-\alpha+1\right)}{\left[\Gamma\left(2 n^{*}+1\right) \Gamma\left(2 n^{\prime \prime}-2 \alpha+1\right)\right]^{1 / 2}} \\
& \Omega_{n \kappa}^{\alpha l}(N)=\left[\frac{[2(k-\alpha)] !}{(2 \kappa) !}\right]_{n^{\prime}=\max (n, \kappa)}^{\frac{1}{2}}\left(2 n^{\prime}\right)^{\alpha} \omega_{n^{\prime} n}^{\alpha l} \omega_{n^{\prime} \kappa}^{\alpha l} \\
& \omega_{n n^{\prime}}^{\alpha l}=(-1)^{n^{\prime}-l-1}\left[\frac{\left(n^{\prime}+l+1\right) !}{(2 n)^{\alpha}\left(n^{\prime}+l+1-\alpha\right) !}\right. \\
& \times F_{n^{\prime}+l+1-\alpha}(n+l+1-\alpha) F_{n^{\prime}-l-1}(n-l-1) \\
& \left.\quad \times F_{n^{\prime}-l-1}\left(2 n^{\prime}\right)\right]^{1 / 2}
\end{aligned}
$$

The $S_{l m}(\theta, \varphi)$ occurring in Eqs. (2) and (3) are the complex $\left(S_{l m} \equiv Y_{l m}\right)$ or real spherical harmonics. We notice that the definition of phases in this work for the complex spherical harmonics $\left(Y_{l m}^{*}=Y_{l-m}\right)$ differs from the Condon-Shortley phases [12] by the sign factor $(-1)^{m}$.

\section{Evaluation of overlap integrals over NISTOs}

The two-center overlap integrals over NISTOs in molecular coordinate system are defined by

$$
\begin{aligned}
& S_{n^{*} l m, n^{*} l^{\prime} m^{\prime}}\left(\zeta, \zeta^{\prime} ; \boldsymbol{R}\right) \\
& \quad=\int \chi_{n^{*} l m}^{*}\left(\zeta, \boldsymbol{r}_{a}\right) \chi_{n^{\prime *} l^{\prime} m^{\prime}}\left(\zeta^{\prime}, \boldsymbol{r}_{b}\right) \mathrm{d} V .
\end{aligned}
$$

Using Eq. (1) we obtain for the two-center overlap integrals over NISTOs the following relations through the two-center overlap integrals with ISTOs [7]:

$$
\begin{aligned}
& S_{n^{*} l m, n^{\prime *} l^{\prime} m^{\prime}}\left(\zeta, \zeta^{\prime} ; \boldsymbol{R}\right) \\
& \quad=\lim _{N, N^{\prime} \rightarrow \infty} \sum_{n^{\prime \prime}=l+1}^{N} \sum_{n^{\prime \prime \prime}=l^{\prime}+1}^{N^{\prime}} V_{n^{*} l, n^{\prime \prime} l}^{\alpha N} V_{n^{\prime *} l^{\prime}, n^{\prime \prime \prime} l^{\prime}}^{\alpha N^{\prime}} \\
& \quad \times S_{n^{\prime \prime} l m, n^{\prime \prime \prime} l^{\prime} m^{\prime}}\left(\zeta, \zeta^{\prime}, \boldsymbol{R}\right),
\end{aligned}
$$

where $\boldsymbol{R}=\boldsymbol{R}_{a b}$ and

$$
\begin{aligned}
& S_{n l m, n^{\prime} l^{\prime} m^{\prime}}\left(\zeta, \zeta^{\prime} ; \boldsymbol{R}\right) \\
& \quad=\int \chi_{n l m}^{*}\left(\zeta, \boldsymbol{r}_{a}\right) \chi_{n^{\prime} l^{\prime} m^{\prime}}\left(\zeta^{\prime}, \boldsymbol{r}_{b}\right) \mathrm{d} V .
\end{aligned}
$$

The analytical relations for two-center overlap integrals over ISTOs have been presented in Refs. [13, 14].
In order to evaluate the two-center overlap integrals over ISTOs in molecular coordinate system, we use the following formula [13]:

$$
\begin{aligned}
& S_{n l m, n^{\prime} l^{\prime} m^{\prime}}\left(\zeta, \zeta^{\prime} ; \boldsymbol{R}\right) \\
& =\sum_{\lambda=0}^{\min \left(l, l^{\prime}\right)} T_{l m, l^{\prime} m^{\prime}}^{* \lambda}(\theta, \varphi) S_{n l \lambda, n^{\prime} l^{\prime} \lambda}\left(\zeta, \zeta^{\prime} ; R\right),
\end{aligned}
$$

where $T_{l m, l^{\prime} m^{\prime}}^{\lambda}(\theta, \varphi)$ and $S_{n l \lambda, n^{\prime} l^{\prime} \lambda}\left(\zeta, \zeta^{\prime} ; R\right)$ are the Guseinov rotational angular coefficients and overlap integrals over ISTOs in lined-up coordinate systems, respectively (see Refs. [15, 16]). For the calculation of $S_{n l \lambda, n^{\prime} l^{\prime} \lambda}\left(\zeta, \zeta^{\prime} ; R\right)$ we use the Löwdin $\alpha$ radial function [10] in the following form [17-22]:

$$
\begin{aligned}
& S_{n l \lambda, n^{\prime} l^{\prime} \lambda}\left(\zeta, \zeta^{\prime} ; R\right)=N_{n l, n^{\prime} l^{\prime} \lambda}(-1)^{l^{\prime}+\lambda}\left(\frac{\zeta}{\zeta^{\prime}}\right)^{n+1 / 2} \\
& \quad \times \sum_{i=0}^{n^{\prime}+l^{\prime}+l} \sum_{j=0}^{n^{\prime}+l}(n-l+j) ! C_{l}^{n^{\prime} l^{\prime} \lambda}(i, j) \\
& \quad \times\left(\zeta^{\prime} R\right)^{n-2 l-l^{\prime}+i+j} \\
& {\left[\mathrm{e}^{-\zeta^{\prime} R}\left(\frac{(-1)^{j}}{\left(R\left(\zeta-\zeta^{\prime}\right)\right)^{n-l+j+1}}-\frac{1}{\left(R\left(\zeta+\zeta^{\prime}\right)\right)^{n-l+j+1}}\right)\right.} \\
& \quad+\mathrm{e}^{-\zeta R} \sum_{k=0}^{n-l+j} \frac{(-1)^{i}}{(n-l+j-k) !}\left(\frac{1}{\left(R\left(\zeta+\zeta^{\prime}\right)\right)^{k+1}}\right. \\
& \left.\left.\quad-\frac{(-1)^{j}}{\left(R\left(\zeta-\zeta^{\prime}\right)\right)^{k+1}}\right)\right],
\end{aligned}
$$

where

$$
\begin{aligned}
& N_{n l, n^{\prime} l^{\prime} \lambda}=(-1)^{\lambda} 2^{n^{\prime}+n} \\
& \quad \times\left(\frac{(2 l+1)\left(2 l^{\prime}+1\right)\left(l^{\prime}+\lambda\right) !(l-\lambda) !}{(2 n) !\left(2 n^{\prime}\right) !(l+\lambda) !\left(l^{\prime}-\lambda\right) !}\right)^{1 / 2} .
\end{aligned}
$$

Here the coefficients $C_{l^{\prime}}^{n l \lambda}(i, j)$ can be calculated by using Sharma's analytical formulae [23-27]. For the quick calculations, in our earlier study, the Sharma analytical formula has been expressed in terms of binomial coefficients. Thus, the Sharma analytical formula for the coefficients $C_{l^{\prime}}^{n l m}(i, j)$ occurring in Löwdin $\alpha$ radial function becomes [28]:

$$
C_{l^{\prime}}^{n l \lambda}(i, j)=\left\{\begin{array}{l}
\left(n+l+2 l^{\prime}-i-j\right) ! \sum_{\mu=0}^{\min \left\{i / 2, l+l^{\prime}\right\}} \sum_{\nu=0}^{\min \left\{j / 2, l+l^{\prime}-\mu\right\}} F_{j-2 \nu}\left(n+l+2 l^{\prime}-2 \nu-i\right) \\
\quad \times F_{i-2 \mu}\left(n+l+2 l^{\prime}-2 \mu-2 \nu\right) B_{\mu \nu}^{l l^{\prime} \lambda} \text { for } i+j \leq n+l-\lambda+2 l^{\prime}, \\
0 \quad \text { for } i+j>n+l-\lambda+2 l^{\prime},
\end{array}\right.
$$


TABLE I

The comparative values of two-center overlap integrals with ISTOs and NISTOs obtained from Eq. (8) for $\alpha=-1, \alpha=-3$ and $N=N^{\prime}=20$.

\begin{tabular}{c|c|c|c|c|c|c|c|c|c|c|c|c|c}
\hline \hline$n$ & $l$ & $m$ & $\zeta$ & $n^{\prime}$ & $l^{\prime}$ & $m^{\prime}$ & $\zeta^{\prime}$ & $p$ & $\theta$ & $\varphi$ & $\begin{array}{c}\alpha=-1 \text { for } \\
\text { Eq. (9) in [11] }\end{array}$ & $\begin{array}{c}\alpha=-1 \\
\text { for Eq. (8) }\end{array}$ & $\begin{array}{c}\alpha=-3 \\
\text { for Eq. (8) }\end{array}$ \\
\hline 2.3 & 0 & 0 & 7.5 & 2.8 & 0 & 0 & 2.5 & 6 & 0 & 0 & 0.254682 & 0.254677 & 0.254594 \\
2.9 & 1 & 0 & 6 & 2.6 & 1 & 1 & 4 & 8 & 30 & 45 & 0.089117 & 0.089117 & 0.089117 \\
3.1 & 2 & 2 & 15 & 3.5 & 2 & 2 & 10 & 2.5 & 120 & 135 & 0.300441 & 0.300441 & 0.300441 \\
3 & 2 & 2 & 15 & 3 & 2 & 2 & 10 & 2.5 & 120 & 135 & 0.255396 & 0.255396 & 0.255396 \\
4.5 & 3 & 2 & 25 & 3 & 2 & 2 & 17 & 4.2 & 150 & 180 & -0.257333 & -0.257333 & -0.257333 \\
4 & 3 & 1 & 50 & 4.7 & 2 & 1 & 40 & 1.4 & 150 & 180 & -0.21316 & -0.21316 & -0.21316 \\
4.6 & 3 & 3 & 26 & 4.3 & 3 & 3 & 24 & 5.1 & 180 & 225 & 0.345451 & 0.345451 & 0.345451
\end{tabular}

TABLE II

The values of overlap integrals with ISTOs and NISTOs obtained from Eq. (8) for $\alpha=-3, \alpha=-4$ and $N=N^{\prime}=30$.

\begin{tabular}{c|c|c|c|c|c|c|c|c|c|c|c|c}
\hline \hline$n$ & $l$ & $m$ & $\zeta$ & $n^{\prime}$ & $l^{\prime}$ & $m^{\prime}$ & $\zeta^{\prime}$ & $R$ & $\theta$ & $\varphi$ & $\begin{array}{c}\alpha=-3 \\
\text { for Eq. (8) }\end{array}$ & $\begin{array}{c}\alpha=-4 \\
\text { for Eq. (8) }\end{array}$ \\
\hline 2.1 & 1 & 0 & 0.7 & 2.3 & 0 & 0 & 0.3 & 0.2 & 50 & 120 & 0.00020027 & 0.00019882 \\
3.2 & 2 & 0 & 1.3 & 3.6 & 2 & 1 & 0.7 & 2.5 & 20 & 36 & 0.016633 & 0.016633 \\
4.3 & 3 & 2 & 2.2 & 4.1 & 2 & 2 & 1.2 & 3.2 & 60 & 30 & -0.034904 & -0.034904 \\
5.1 & 4 & 2 & 1.5 & 4.8 & 3 & 2 & 0.5 & 2 & 120 & 60 & -0.027229 & -0.027229 \\
5.6 & 3 & 3 & 1.5 & 5.3 & 4 & 3 & 0.5 & 2 & 45 & 120 & -0.091578 & -0.091578 \\
6 & 4 & 4 & 23 & 5 & 4 & 4 & 27 & 0.5 & 150 & 60 & -0.0062132 & -0.0062132 \\
6.5 & 5 & 3 & 46 & 5.7 & 4 & 3 & 42 & 0.16 & 125 & 180 & 0.090123 & 0.090123 \\
7.2 & 6 & 5 & 46 & 6.1 & 4 & 4 & 42 & 0.16 & 135 & 150 & -0.021633 & -0.021633 \\
\end{tabular}

where

$$
\begin{aligned}
& B_{\mu \nu}^{l l^{\prime} \lambda}=F_{\mu}\left(\mu+\nu-l^{\prime}-1 / 2\right)\left(\nu-l^{\prime}-1 / 2\right) ! \\
& \quad \times\left(l^{\prime}-\nu-1 / 2\right) ! \sum_{s=0}^{l-\lambda} F_{s}(l-\lambda) F_{l+\lambda-s}\left(l^{\prime}+\lambda\right) \\
& \quad \times F_{l+l^{\prime}-\mu-\nu}\left(l^{\prime}-\mu+s-1 / 2\right) F_{\nu}(s-l+\nu-1 / 2) .
\end{aligned}
$$

The quantity $F_{n}(m)=\frac{n !}{m !(n-m) !}$ in Eqs. (6), (13) and (14) are the binomial coefficients.

\section{Numerical results and discussion}

An efficient calculation algorithm is presented for the two-center overlap integrals over NISTOs in molecular coordinate system by using Guseinov's one-center expansion formulae and Löwdin $\alpha$ radial function. The proposed algorithm is implemented numerically using a computer program, and its convergence properties are investigated. We performed a program for the calculation of two-center overlap integrals over NISTOs and ISTOs in molecular coordinate system. All calculations were performed on Mathematica 7.0 international mathematical software. The computational results and liter- ature [11] data of the two-center overlap integrals over NISTOs and ISTOs in molecular coordinate system are shown in Tables I and II for $\alpha=-1, \alpha=-3$ and $\alpha=-4$. As can be seen from the tables, the computation accuracy of computer results is satisfactory.

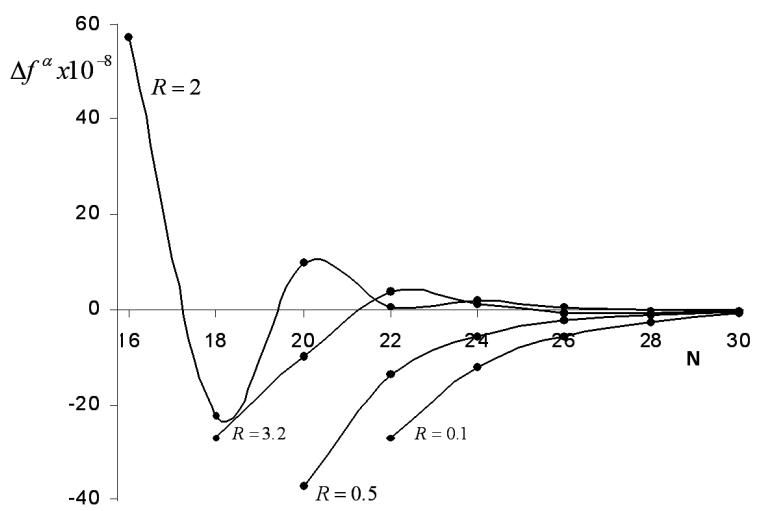

Fig. 1. The convergence of series in Eq. (8) for $\alpha=-1$ with different values of parameter $R$ for two-center overlap integral $S_{3.7,2,2,3.3,2,2}$ in atomic units (a.u.) as a function of the indices $N=N^{\prime}\left(\zeta=5.5, \zeta^{\prime}=2.2, \theta=\right.$ $30^{\circ}, \varphi=300^{\circ}$ ). 


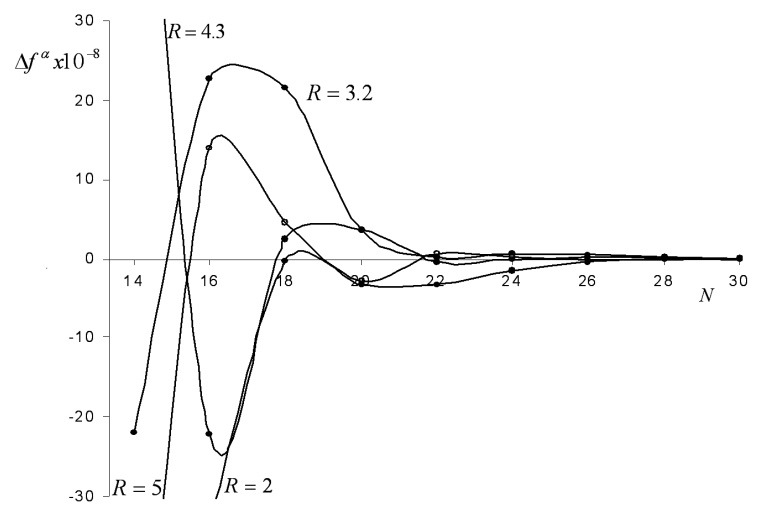

Fig. 2. The convergence of series in Eq. (8) for $\alpha=-3$ with different values of parameter $R$ for two-center overlap integral $S_{3.7,2,2,3.3,2,2}$ in a.u. as a function of the indices $N=N^{\prime}\left(\zeta=5.5, \zeta^{\prime}=2.2, \theta=30^{\circ}, \varphi=300^{\circ}\right)$.

In Figs. 1 and 2 we present the convergence of the series in Eq. (8) for $N=N^{\prime}=30$. Here $N$ and $N^{\prime}$ are the upper limits of the $n^{\prime \prime}$ and $n^{\prime \prime \prime}$, respectively. The series accuracy $\Delta f^{\alpha}=f_{N}^{\alpha}-f_{L}^{\alpha}$ is shown in Figs. 1 and 2, where the quantities $f_{L}^{\alpha}$ are the values of integral for $L<N=N^{\prime}$. As can be seen from Figs. 1 and 2, the convergence for given $\alpha$ is satisfactory for arbitrary values of internuclear distances $R$. Greater accuracy is attainable by the use of more terms of expansions (8). The convolution between Guseinov's one-center expansion formulae and Löwdin $\alpha$ radial function to accurate calculations of the two-center overlap integrals with NISTOs in molecular coordinate system is reported for the first time in the literature. Extensive numerical tests and comparisons with some already existing methods show that the algorithm proposed here is efficient.

\section{Acknowledgments}

One of the authors (E.Ç.) thanks TUBITAK for financial support.

\section{References}

[1] J.C. Slater, Quantum Theory of Atomic Structure, Vol. 2, Mc Graw-Hill, New York 1960.

[2] S.F. Boys, Prog. R. Soc. A 200, 542 (1950).

[3] T. Kato, Commun. Pure Appl. Math. 10, 151 (1957).

[4] S. Agmon, Lectures on Exponential Decay of Solutions of Second-Order Elliptic Equations: Bounds on Eigenfunctions of $\mathrm{N}$-Body Schrödinger Operators, Princeton University Press, Princeton, NJ 1982.

[5] I.I. Guseinov, Int. J. Quantum Chem. 90, 114 (2002).
[6] I.I. Guseinov, B.A. Mamedov, J. Mol. Model. 8, 272 (2002).

[7] I.I. Guseinov, B.A. Mamedov, J. Math. Chem. 43 1527 (2008).

[8] B.A. Mamedov, H. Koç, J. Math. Chem. 44, 365 (2008).

[9] B.A. Mamedov, E. Çopuroğlu, MATCH Commun. Math. Comput. Chem. 61, 553 (2009).

[10] P.O. Löwdin, Adv. Phys. 5, 96 (1956); Rev. Mod. Phys. 32, 328 (1960); Rev. Mod. Phys. 34, 520 (1962).

[11] I.I. Guseinov, B.A. Mamedov, Commun. Theor. Phys. 42, 753 (2004).

[12] E.U. Condon, G.H. Shortley, Theory of Atomic Spectra, Cambridge University Press., London 1965.

[13] I.I. Guseinov, Phys. Rev. A 32, 1864 (1985).

[14] I.I. Guseinov, B.A. Mamedov, J. Mol. Struct. (Theochem) 465, 1 (1999).

[15] I.I. Guseinov, J. Mol. Struct. (Theochem) 343, 173 (1995).

[16] I.I. Guseinov, B.A. Mamedov, Z. Naturforsch. A 62a, 467 (2007).

[17] H.W. Jones, Int. J. Quantum Chem. 18, 709 (1980); 19, 567 (1981); 21, 1079 (1982); 23, 953 (1983); 29, 177 (1986); 41, 749 (1992); 45, 21 (1993); 61, 881 (1997); Phys. Rev. A 30, 1 (1984); 33, 3 (1986); 35, 4 (1987); 30, 2 (1988); 38, 2 (1988); J. Comput. Chem. 12, 1217 (1991); Int. J. Quantum Chem. Symp. 15, 287 (1981).

[18] J.L. Jain, H.W. Jones, C.A. Weatherford, P.E. Hoggan, Int. J. Quantum Chem. 100, 199 (2004).

[19] H.W. Jones, J.L. Jain, Int. J. Quantum Chem. 30, 1257 (1996).

[20] C.A. Weatherford, H.W. Jones, in: Int. Conf. on ETO Multicenter Integrals, Reidel, Dordrecht 1982, p. 152.

[21] H.W. Jones, C.A. Weatherford, Int. J. Quantum Chem. S12, 483 (1978).

[22] H.W. Jones, B. Bussery, C.A. Weatherford, Int. J. Quantum Chem. 32, 693 (1987).

[23] R.R. Sharma, T.P. Das, R. Orbach, Phys. Rev. 155 338 (1967).

[24] R.R. Sharma, J. Math. Phys. 9, 505 (1968).

[25] R.R. Sharma, Phys. Rev. A 13, 2 (1976); 13, 517 (1976).

[26] R.R. Sharma, Int. J. Quantum Chem. 10, 1075 (1976).

[27] N. Suzuki, J. Math. Phys. 25, 1133 (1984); 25 313(E) (1984); 26, 3193 (1985); 28, 4 (1987); 31, 9 (1990); 33, 12 (1992).

[28] B.A. Mamedov, E. Çopuroğlu, J. Math. Chem. 49, 201 (2011). 